Thorax (1970), 25, 499.

\title{
Translocation of the atrial septum
}

\author{
C. R. C. W Y N D H A M ${ }^{1}$ \\ Pathology Department, University of Adelaide, South Australia
}

Translocation of the atrial septum to the left of the mitral valve is a congenital anomaly : the author has not found a similar case previously reported in the English literature. The curious anomaly described here was associated with deformed mitral and tricuspid valves, a posteroinferior atrial septal defect, a persistent left superior vena cava, a hypoplastic aorta, and a short innominate artery. The patient lived to a remarkable age.

\section{CASE REPORT}

No details are available of the antenatal history of the patient, an unmarried white Australian woman. who was born a 'blue baby'. 'Heart disease' or 'coronaries' were responsible for the deaths of her mother at the age of 73 years, her father at 67 years, and six of his siblings between the ages of 40 and 50 years. Since childhood she had had chronic bronchitis and was prone to infections. She worked hard as a dressmaker's machinist and had no cardiac symptoms until the age of 47 years, when she noticed palpitations, nocturnal breathlessness, syncopal attacks, dizziness, and coldness of the extremities of gradual onset. Within two years there were clinical and radiological signs of an enlarging heart (Fig. 1), and there was a precordial systolic murmur transmitted to the left axilla. At the age of 49 years she developed angina of effort and an electrocardiogram showed S-T segment depression in leads V2 to V4 with aggravation by exercise. During the next seven years she gradually developed mild biventricular failure, but at the age of 53 years she claimed to have been 'climbing mountains' without undue distress while on holiday. Ultimately she had to retire because of moderate exertional dyspnoea.

Her terminal admission to hospital at the age of 55 years was with haemoptysis, pleuritic chest pain, inability to speak, and mild right hemiparesis. She was conscious but had a motor aphasia and a rightsided pyramidal lesion involving the face, arm, and leg. There was central and peripheral cyanosis and finger clubbing. The pulse rate was 78 per minute, and there were multiple extrasystoles. The blood pressure was $120 / 60 \mathrm{~mm}$. Hg. There was mild elevation of the jugular venous pressure, a laterally displaced thrusting apex beat, and a right ventricular impulse in the left parasternal area. She had a presystolic triple rhythm, a systolic murmur at the left

1Present address: Repatriation General Hospital, Daws Road. Daw Park, South Australia, 5041 sternal edge, and signs of consolidation at both lung bases. Initial investigations disclosed a leukoerythroblastic blood picture and a reticulocytosis of $5.0 \%$ without anaemia, and there was considerable elevation of the serum alkaline phosphatase and glutamicoxalacetic transaminase.

An electrocardiogram showed the mean axis in the frontal plane to be $-136^{\circ}$ and there was clockwise rotation in the horizontal plane. It satisfied Milnor's and Roman's criteria for right ventricular hypertrophy (Fig. 2).

Despite broad-spectrum antibiotic therapy, diuresis, and digitalization, she died after cardiac arrest during the passage of a naso-gastric tube on the third day.

\section{NECROPSY}

The body weighed $40 \mathrm{~kg}$. and the heart $440 \mathrm{~g}$. From the external aspect, there was a persistent left superior vena cava draining the left internal jugular and subclavian veins and showing no vestige of an innominate vein. The innominate artery was greatly foreshortened to about $0.5 \mathrm{~cm}$. in length and the aorta was uniformly hypoplastic, measuring $1.3 \mathrm{~cm}$. in diameter. The right atrium received all three caval veins and the coronary sinus, and was itself greatly dilated and hypertrophied. The interatrial septum was placed very obliquely, being attached superiorly in the normal position, but inferiorly to the atrioventricular ring at the left of the mitral valve, so that the right atrium led directly into both atrioventricular canals (Fig. 3). The left atrium was also hypertrophied and a little dilated and received all four pulmonary veins. It communicated with the right atrium via an oval atrial septal defect, $1 \mathrm{~cm}$. in diameter, $0.5 \mathrm{~cm}$. above the mitral valve ring, posteriorly. Otherwise the atrial septum appeared normal, containing a rather anteriorly placed fossa ovalis which exhibited flap-valve probe patency 


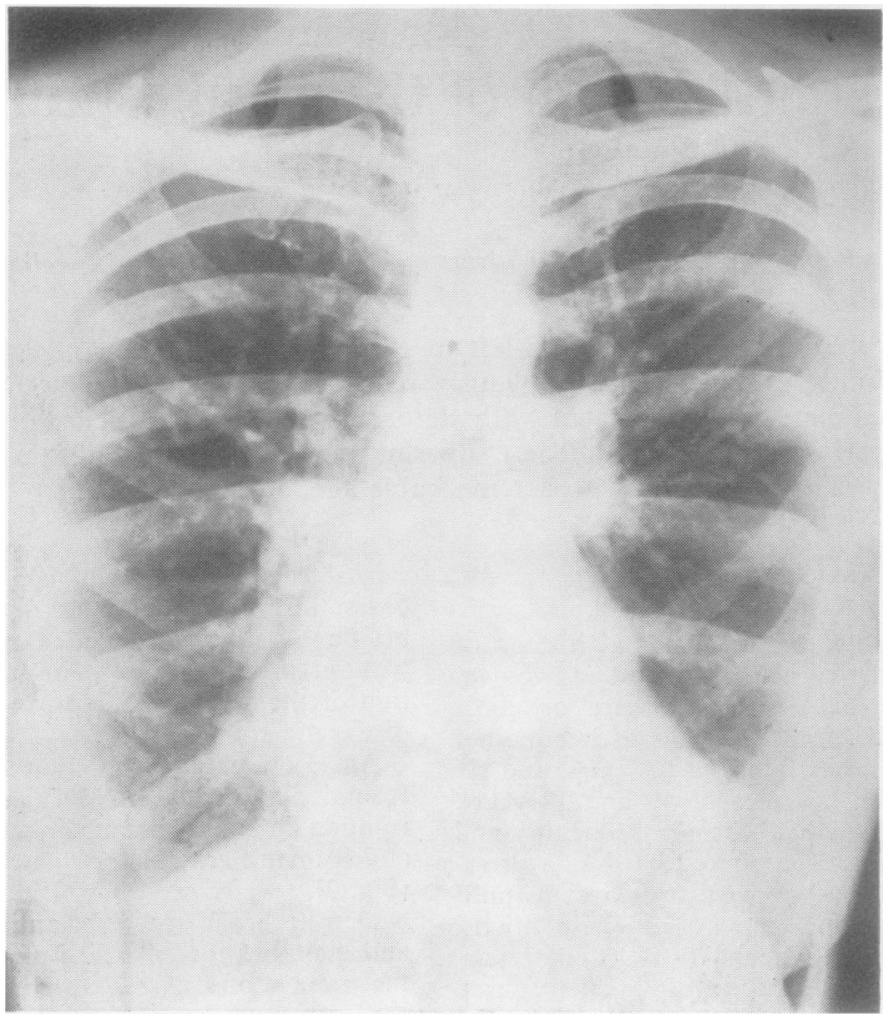

FIG. 1. Postero-anterior chest radiograph.
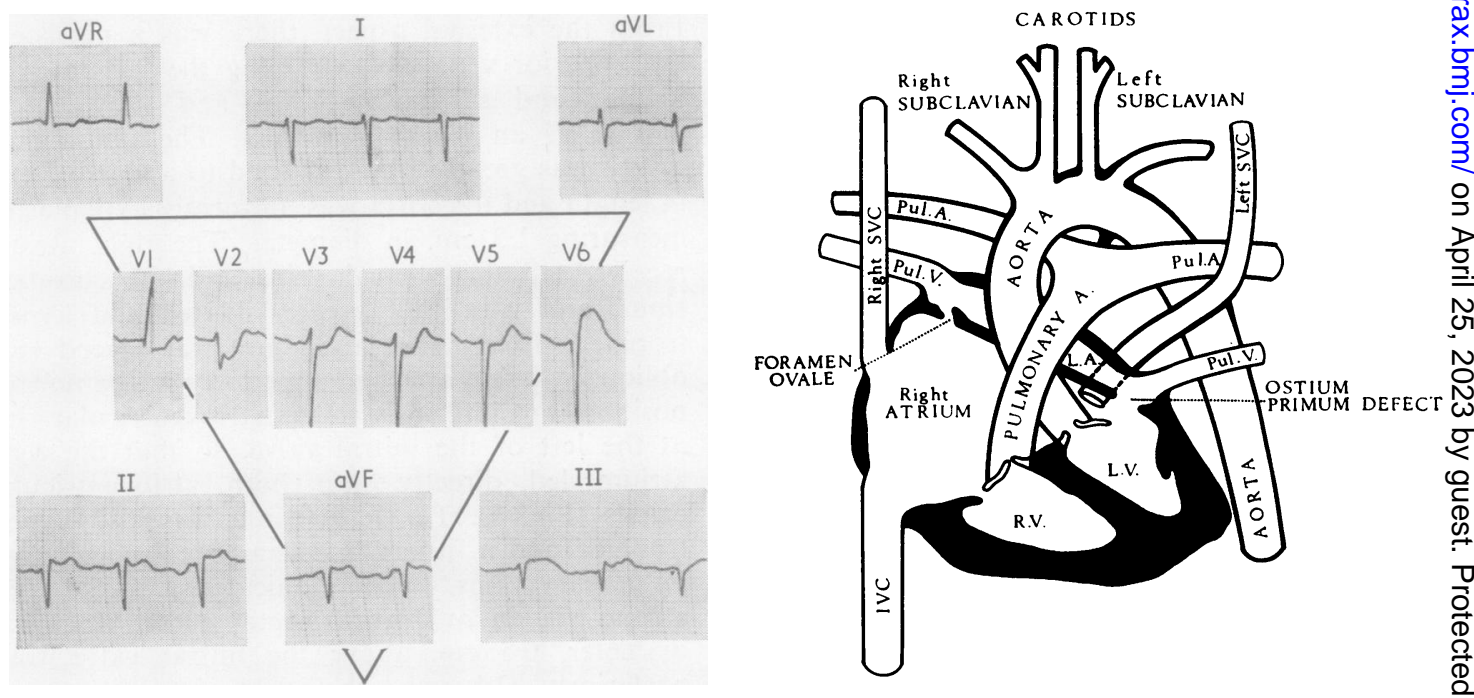

FIG. 2. Electrocardiogram made two days before death.

FIG. 3. Schematic representation of the cardiovascular findings at necropsy. 


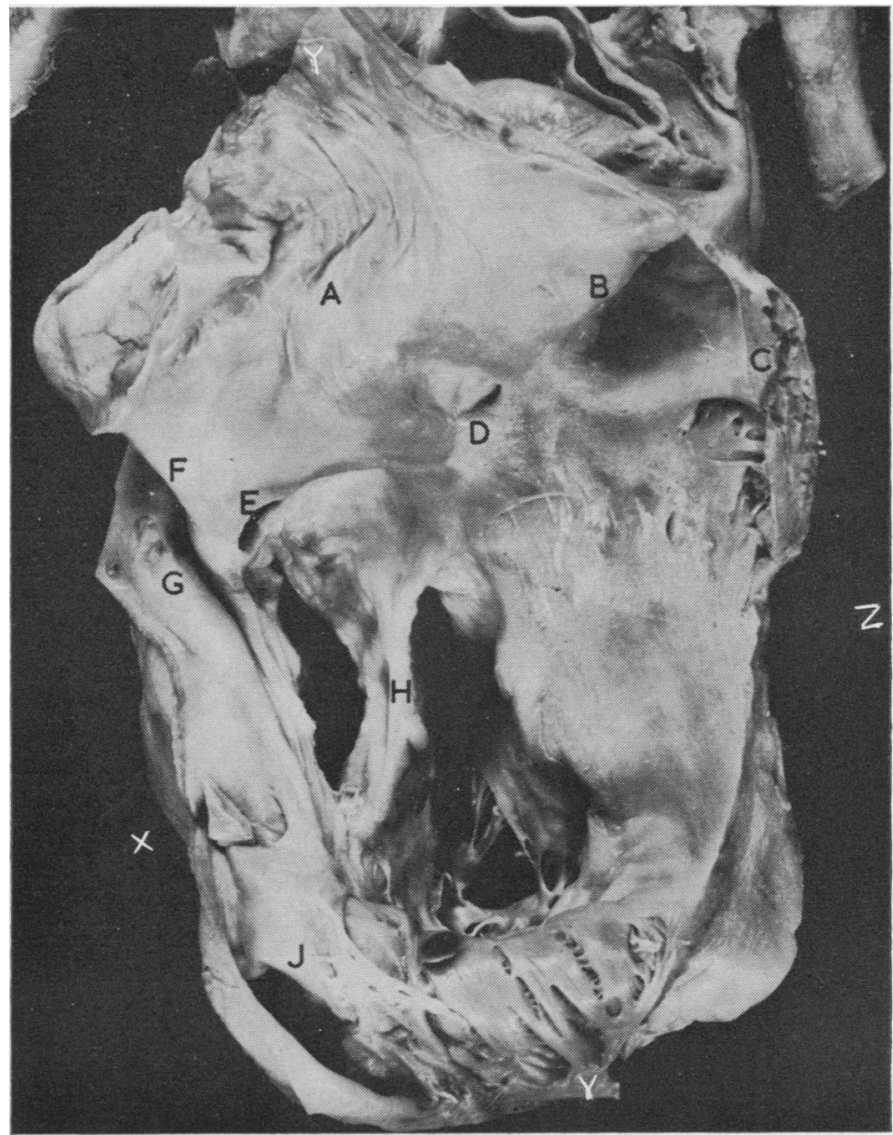

FIG. 4. Postero-anterior view of right atrium opened along the line $X Y Z$. A. Right aspect of the atrial septum, B. Lip of the right superior vena caval orifice; $C$. Right atrial appendage; $D$. Probe-patent foramen ovale; E. Postero-inferior atrial septal defect; $F$. Entry into the right atrium of the persistent superior vena cava; $G$. Orifice of the coronary sinus; $H$. Fibrous trigone-cleft mitral valve to the left and deformed tricuspid valve to the right; $J$. Chiari net and inferior vena caval orifice.

(Fig. 4). There was a small Chiari net adjacent to the inferior vena caval orifice. Both atrioventricular valves showed deficiencies of their septal cusps, that of the mitral (anterior cusp) being represented by a thickened arcuate fold concave downwards, and that of the tricuspid by a small fibrous tag, $0.5 \mathrm{~cm}$. in diameter, attached to the fibrous trigone. The right ventricle was hypertrophied to $0.9 \mathrm{~cm}$. in thickness (Fig. 5) ; the left ventricular cavity was small and the wall measured $1.5 \mathrm{~cm}$. in thickness. There were no demonstrable interventricular communications, and the ductus arteriosus was closed. The pulmonary and aortic valves and the main trunks of the coronary arteries were normal. The pulmonary trunk was of normal dimensions, but recent and old antemortem thrombi filled medium-sized arteries in the left upper lobe and large and medium-sized arteries to the lower lobes of both lungs which contained multiple old and recent infarcts. There was thrombosis of the para-vaginal plexus and small adherent thrombi in the right atrial appendage, no doubt accounting for recurrent pulmonary embolism.

The liver and spleen were greatly enlarged and almost totally infarcted. Thrombo-emboli were impacted in the main hepatic and splenic arteries and in a branch of the superior mesenteric artery, 


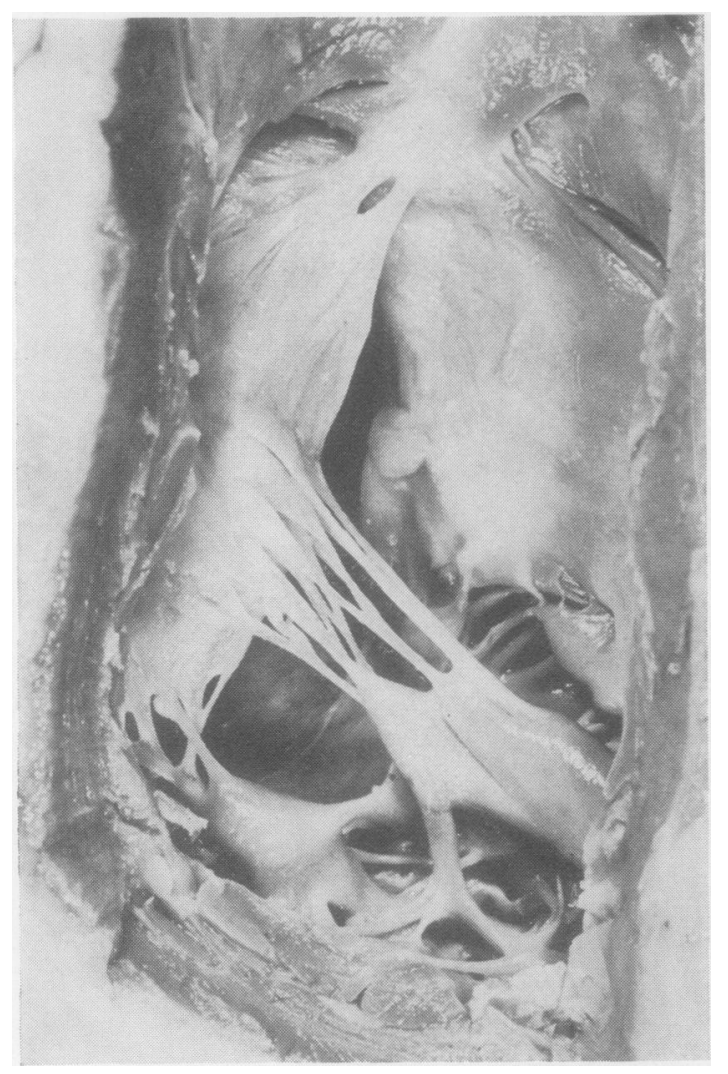

FIG. 5. Ventricular aspect of the tricuspid valve, showing the deformities, seen through a window cut in the hypertrophied anterior wall of the right ventricle.

no doubt having travelled paradoxically through the right atrium and left ventricle.

Other evidence for paradoxical embolism consisted of: (1) a focal embolic glomerulonephritis with platelet thrombi in many isolated glomerular loops, with organizing thrombus in an interlobar renal artery; (2) platelet thrombi in small vessels in the left corona radiata with histological cortical infarction; (3) small, old, gross softenings in the right lobe of the cerebellum and in the left subthalamic region; and (4) multiple micro-infarcts, mainly perivascular and of varying ages, in the myocardium of all chambers, associated with marked endocardial fibro-elastosis and small mural thrombi. Arteries in the lungs showed organizing and recanalizing thrombi, moderate thickening, fibroelastosis, and patchy atherosclerosis of elastic arteries, prominent bronchial arteries, but little change in the small muscular arteries and arterioles.

\section{DISCUSSION}

The lack of proper cardiovascular investigation in this case unfortunately prevents any conclusions $\vec{O}$ as to intracardiac pressure-flow dynamics, but on a $\overrightarrow{\vec{A}}$ superficial consideration of the complex anomaly $\vec{\omega}$ involved it seems remarkable that the patient lived as long and as symptom-free as she did, and that $x$ histological evidence of pulmonary hypertension or was so slight, and consistent with recurrent + thrombo-embolism per se.

The genesis of the cardiac anomaly remains? uncertain but presumably represents some variant of an endocardial cushion defect, with a low atrial $\vec{c}$ septal defect, cleft anterior mitral valve leaflet, and deformed septal tricuspid valve leaflet, but without ventricular septal defect, whose association with deformed septal tricuspid valve leaflet, caus-. ing left ventricular-right atrial shunt, is well recog. nized. In the present case any shunt between these two chambers would have been due to the mitralo deformity and the displacement of the atrial septum. Persistent left superior vena cava has been $\mathbb{\complement}$ reported to enter the left atrium in association with $\overrightarrow{\vec{O}}$ a postero-inferior atrial septal defect and absence 3 of the coronary sinus, a complex attributed to failure of development of the left atriovenous foldo (Raghib, Ruttenberg. Anderson, Amplatz, Adams, and Edwards, 1965). Although the site of the atrialo septal defect in the present case corresponds to their description, the left caval vein entered theo right atrium and a coronary sinus was present However, the longevity of this patient may per-o haps be attributed to the relatively benign haemodynamic situation found in such patients, namelyo a left-to-right shunt at atrial level and mild arterial ${ }_{\triangle}$ oxygen desaturation without pulmonary hyper-0 tension. In the absence of studies during life these thoughts are admittedly speculative. Finally, the $e^{\mathrm{r}}$ age of the patient, the presence of a well-formed $N$ foramen ovale in the atrial septum, and the absence of Mongolism dispose of the remote pos-o sibility that the anomaly was cor triloculare bi ventriculare with a separate chamber draining the pulmonary veins.

Whatever the haemodynamic situation presento for the greater part of the patient's life, cardiaco decompensation was undoubtedly due to a gradua $\overrightarrow{\mathbb{B}}$ rise in right ventricular pressure secondary toD recurrent pulmonary embolism, and death was due to the combined effect of the latter and hepatic infarction. 
My thanks are due to Dr. T. D. Finey and to Dr. H. R. Gilmore for permission to publish the clinical record, and to Professor J. S. Robertson and Dr. P. S. Hetzel for their helpful advice and criticism. Miss H. Fuller and Mr. D. N. Caville prepared the drawings and photographs.

\section{REFERENCES}

Raghib, G., Ruttenberg, H. D., Anderson, R. C., Amplatz, K., Adams, P., and Edwards, J. E. (1965). Termination of left superior vena cava in left atrium, atrial septal defect and absence of coronary sinus. A developmental complex. Circulation, 31, 906. 